\title{
Evaluación de los recursos turísticos naturales del municipio de San Pedro Lagunillas, Nayarit, México, a partir de la metodología multicriterio
}

Avaliação dos recursos turísticos naturais do município de San Pedro Lagunillas, Nayarit, México, a partir da metodologia multicritério Evaluation of natural tourism resources of the municipality of San Pedro Lagunillas, Nayarit, Mexico, from the multi-criteria methodology http://dx.doi.org/10.18472/cvt.16n3.2016.0987

Daniela Arciniega Camarena 〈 arciniega219o@gmail.com > Universidad Autónoma del Estado de México (UAEMex), Cidade do México, México.

Maribel Osorio García 〈maribelosorio2@gmail.com >

Universidad Autónoma del Estado de México (UAEMex), Cidade do México, México.

Hector Hugo Regil García 〈regil_hh@yahoo.com.mx >

Universidad Autónoma del Estado de México (UAEMex), Cidade do México, México.

CRONOLOGIA DO PROCESSO EDITORIAL

Recebimento do artigo: 31-out-2014

Aceite: 22-ago-2016

FORMATO PARA CITAÇÃO DESTE ARTIGO

ARCINIEGA, D.; OSORIO, M.; REGIL, H. H. Evaluación de los Recursos Turísticos Naturales del Municipio de San Pedro Lagunillas, Nayarit, México, a partir de la metodología multicriterio. Caderno Virtual de Turismo. Rio de Janeiro, v. 16, n. 3, p. 43-6o, dez. 2016.

REALIZAÇÃO

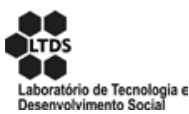

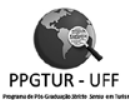

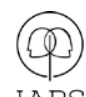

APOIO INSTITUCIONAL

EDIÇÃO

PATROCÍNIO

COPPE

UFR]

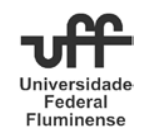

EDITORค 


\section{RESUMO}

O turismo de natureza é considerado no México como uma alternativa de diversificação turística, por meio do qual é possível valorizar os recursos naturais com potencial recreativo-turístico. É o caso do município de San Pedro Lagunillas (SPL) no estado de Nayarit, no México, que tem um clima quente e corpos de água que moldaram locais suscetíveis de belezas naturais para o desenvolvimento turístico. Então, o objetivo deste trabalho é avaliar o potencial dos recursos naturais no município de SPL, a fim de propor um produto turístico focado no turismo de natureza. A metodologia envolveu a integração de um inventário dos recursos e a respectiva avaliação desses recursos, a escolha da metodologia de avaliação multicritério (EMC) como o procedimento quantitativo relevantes para a classificação e priorização. Verificou-se que o Parque Ecológico Luis Donaldo Colosio foi o recurso melhor avaliado, principalmente pelo peso dos seus valores extrínsecos.

Palavras-chave: Turismo de natureza. Avaliação dos recursos turísticos. Inventários. Avaliação multicritério.

\section{ABSTRACT}

Nature tourism is considered in Mexico as an alternative tourism diversification through which it is possible to value natural resources with recreational - tourism potential. Such is the case of the municipality of San Pedro Lagunillas (SPL) in the Nayarit State, Mexico, which has a warm climate and water bodies that have shaped natural beauty susceptible sites for tourist development. Therefore, the objective of this paper is to assess the potential of natural resources in the municipality of SPL, in order to propose a tourism product focused nature tourism. The methodology involved the integration of an inventory of resources and the respective evaluation of them, choosing multi-criteria evaluation methodology (EMC) as the relevant quantitative procedure for classification and prioritization. It was found that the Ecological Park Luis Donaldo Colosio was the best evaluated resource mainly to the weight of their extrinsic values.

Keywords: Natural tourism. Evaluation of resources tourism. Inventory. Multicriteria evaluation.

\section{RESUMEN}

El turismo de naturaleza se considera en México como una alternativa de diversificación turística a través del cual es posible poner en valor recursos naturales con potencialidad recreativo-turística. Tal es el caso del municipio de San Pedro Lagunillas (SPL), en el Estado de Nayarit, México, el cual cuenta con un clima cálido y cuerpos de agua que han conformado sitios de belleza natural suceptibles de aprovechamiento turístico. Por ello, el objetivo del presente documento es evaluar la potencialidad de los recursos naturales del municipio de $S P L$, con la finalidad de proponer un producto turístico enfocado al turismo de naturaleza. La metodología aplicada consistió en la integración de un inventario de recursos y la evaluación respectiva de los mismos, eligiendo a la metodología de evaluación multicriterio (EMC) como el procedimiento cuantitativo pertinente para su clasificación y jerarquización. Se encontró que el Parque Ecológico Luis Donaldo Colosio fue el recurso mejor evaluado debido principalmente al peso de sus valores extrínsecos.

Palabras clave: Turismo de naturaleza. Evaluación de recursos turísticos. Inventarios. Evaluación multicriterio. 


\section{Introdução}

El turismo de naturaleza ha sido objeto de atención en México, con el interés de fortalecer este segmento para diversificar su oferta de productos turísticos (Gobierno de la República, 2013; SECTUR, 2002), dado que es uno de los factores mejor calificados para el país en el ranking de competitividad mundial que emite el Foro Económico Mundial (World Economic Forum, 2015), representándole una importante ventaja competitiva.

Así entonces, varios estados del país le han apostado a poner en valor sus recursos naturales potenciales localizados al interior del territorio, una vez que gran parte de los recursos costeros en México se encuentran en una etapa de agotamiento. Entre los estados interesados en esta alternativa, se encuentra el estado de Nayarit, que se limita al poniente con el océano Pacífico, y al norte, oriente y sur tiene colindancia con los estados de Sinaloa, Durango, Zacatecas y Jalisco, que concentran una importante población en el país, particularmente el último de los mencionados. El estado de Nayarit alberga una población de 1,084, 979 habitantes (INEGI, 2010), dedicada a la agricultura, ganadería, pesca y turismo como las actividades económicas mas relevantes en el estado; se divide en 20 municipios, siendo su capital la ciudad de Tepic. Particularmente, el turismo ha ido creciendo en la región, debido a la dinámica que se presenta en el corredor turístico Puerto Vallarta- Rivera Nayarit, uno de los megaproyectos de sol y playa impulsados por el gobierno federal dirigido a turistas extranjeros fundamentalmente, en desatención del turismo doméstico que es numeroso en esa parte del país.

El municipio de San Pedro Lagunillas se ubica en el sur del Estado de Nayarit, localizado entre las coordenadas $20^{\circ} 59^{\prime}$ y $21^{\circ} 20^{\prime}$ de latitud norte y los $104^{\circ} 37^{\prime}$ y $104^{\circ} 54^{\prime}$ de longitud oeste, con una extensión geográfica de $446.25 \mathrm{Km} 2$-lo cual representa el 1.88\% del Estado- en la que se asienta una población total de 7,510 habitantes (INEGI, 2010) en ocho comunidades además de la cabecera municipal: Amado Nervo, Coastecomate, Cerro Pelón, Tequilita, Tepetiltic, Las Guasimas, Colonia Puerta del Rio, Milpillas; cuya ocupación principal se concentra en la agricultura y la ganadería. El municipio posee un rico sistema hidrológico, conformando lagunas y ríos en un territorio de clima cálido subhúmedo y semicálido-húmedo que registra una temperatura media anual de $20.4{ }^{\circ} \mathrm{C}$, el cual favorece el disfrute de diversos sitios de belleza natural. Cuenta además con una ubicación excelente para el desarrollo turístico dado que es paso obligado para los turistas que viajan hacia el destino turístico tradicional de Puerto Vallarta, localizado a $175 \mathrm{~km}$. de la ciudad de Guadalajara y a $57 \mathrm{~km}$. de la ciudad de Tepic (H. Ayuntamiento de San Pedro Lagunillas, 2007). De ahí la pertinencia de desarrollar productos turísticos dirigidos al turismo nacional, que permitan el aprovechamiento de los recursos naturales con belleza escénica y potencialidad recreativa para la práctica del turismo alternativo.

Es importante señalar que en la literatura científica ha sido sumamente cuestionada la forma en que se ha hecho uso de los recursos y espacios naturales para las actividades turísticas debido, entre otros factores, a la ineficiente localización de su oferta de servicios (GARZON Y ARIAS, 2008) a los limitados criterios de zonificación (BOULLÓN, 2006) y la inadecuada gestión de los planes de manejo de las áreas naturales (TORRE Y BOSCHI, 2004), por lo que a partir de considerar que el uso de los recursos naturales debe estar fundamentado en un proceso de planificación estratégica que guíe su aprovechamiento con criterios de sustentabilidad (OSORIO Et al., 2009; RAMÍREZ Y OSORIO, 2007 Y 2008), es necesario desarrollar en el municipio un proceso de planificación que contemple en primera instancia una evaluación de los recursos naturales para desvelar su potencialidad y concretar una propuesta de productos turísticos alternativos pertinentes y con calidad. 
De lo anterior se desprende que el objetivo del presente documento es evaluar la potencialidad de los recursos naturales del municipio de SPL, con la finalidad de proponer un producto turístico enfocado al turismo de naturaleza. Para ello el texto se estructura en cuatro apartados: un marco conceptual-metodológico que refiere los conceptos y metodologías más aplicados para la evaluación de los recursos turísticos; un segundo apartado en el que se expone y aplica la metodología de evaluación multicriterio elegida para la valoración de los recursos naturales del municipio en estudio; un tercer apartado que presenta la propuesta de creación de un producto turístico de aventura para el aprovechamiento de los recursos naturales evaluados; y un último apartado que refiere las conclusiones de la investigación.

\section{Marco conceptual-metodológico}

Los estudios de evaluación y potencialidad de recursos turísticos cuentan con cierta trayectoria en Latinoamérica, desde que en la década de los años setentas la Organización Mundial del Turismo (2001) y la Organización de los Estados Americanos (OEA, 2009) difundieron modelos de evaluación acordes a las características de los países de la región, habiendo sido aplicados por algunas naciones como Colombia y Perú. En el contexto académico, los procesos de planificación turística elaborados por Bote (2002) y Godfrey y Clarke (2002), han aportado claridad para distinguir que una evaluación de recursos turísticos es un proceso que comprende básicamente dos fases: la elaboración de un inventario de los recursos potenciales localizados en el espacio geográfico a evaluar; y la evaluación de los recursos inventariados, la cual puede elaborarse desde una perspectiva cuantitativa (como la de Bote, 2002), o una perspectiva cualitativa (como la de Godfrey y Clark).

La Organización Mundial del Turismo OMT (2001), define al inventario como el recuento de atractivos turísticos, es decir un estado integrado de todos los recursos que existen en el país, región o zona a la que se refiera. Así, se puede decir que un inventario turístico es aquel instrumento en el que se registran datos generales como ubicación, accesibilidad, calidad del entorno, entre otros, de los recursos naturales y culturales susceptibles de aprovechamiento turístico en un determinado espacio. Desde el punto de vista turístico un inventario permite una clasificación de los recursos existentes en un lugar determinado, los datos que se recaban ayudan a identificar el tipo de turismo que atraería y los servicios que se pueden ofrecer para su disfrute.

Entre las metodologías de inventarios y de evaluación aplicadas en México se refieren la de Oscos (1990) y la de SECTUR (2009a y b) ambas dirigidas a todo tipo de recursos, tanto culturales como naturales; las metodologías de Zamorano (2002) y la de Franco et al. (2009), son propias para su aplicación a los recursos naturales, aunque la primera sólo considera la fase de inventario. En la tabla 1 se presentan las variables de análisis que registra cada una de las metodologías mencionadas.

Tabla 1 - Variables de Análisis de las Metodologías de Inventario y Evaluación de Recursos Turísticos.

\begin{tabular}{|l|l|l|l|l|l|l|l|l|}
\hline \multirow{2}{*}{ VARIABLES } & \multicolumn{5}{|c|}{ METODOLOGIAS } \\
\cline { 2 - 8 } & OMT & OEA & OSCOS & ZAMORANO & SECTUR & BOTE & $\begin{array}{l}\text { GODFREY } \\
\text { Y CLARKE }\end{array}$ & MULTICRITERO \\
\hline $\begin{array}{l}\text { Datos } \\
\text { generales }\end{array}$ & & & & & & & \\
\hline Localización & & & & & & & \\
\hline
\end{tabular}




\begin{tabular}{|c|c|c|c|c|c|c|c|c|}
\hline \multirow{2}{*}{ VARIABLES } & \multicolumn{8}{|c|}{ METODOLOGIAS } \\
\hline & OMT & OEA & oscos & ZAMORANO & SECTUR & BOTE & $\begin{array}{l}\text { GODFREY } \\
\text { Y CLARKE }\end{array}$ & MULTICRITERO \\
\hline \multicolumn{9}{|l|}{$\begin{array}{l}\text { Condiciones } \\
\text { generales }\end{array}$} \\
\hline \multicolumn{9}{|l|}{ Accesibilidad } \\
\hline \multicolumn{9}{|l|}{$\begin{array}{l}\text { Tiempo de } \\
\text { llegada }\end{array}$} \\
\hline \multicolumn{9}{|l|}{$\begin{array}{l}\text { Calidad del } \\
\text { entorno }\end{array}$} \\
\hline \multicolumn{9}{|l|}{$\begin{array}{l}\text { Periodo de } \\
\text { visita }\end{array}$} \\
\hline \multicolumn{9}{|l|}{$\begin{array}{l}\text { Costo de } \\
\text { entrada }\end{array}$} \\
\hline \multicolumn{9}{|l|}{$\begin{array}{l}\text { Tiempo de } \\
\text { recorrido }\end{array}$} \\
\hline \multicolumn{9}{|l|}{$\begin{array}{l}\text { Tipo de } \\
\text { visitante }\end{array}$} \\
\hline \multicolumn{9}{|l|}{$\begin{array}{l}\text { Nivel de } \\
\text { afluencia }\end{array}$} \\
\hline \multicolumn{9}{|l|}{$\begin{array}{l}\text { Época propicia } \\
\text { para visitar el } \\
\text { recurso }\end{array}$} \\
\hline \multicolumn{9}{|l|}{$\begin{array}{l}\text { Horario de } \\
\text { visita }\end{array}$} \\
\hline \multicolumn{9}{|l|}{$\begin{array}{l}\text { Actividades } \\
\text { desarrolladas }\end{array}$} \\
\hline \multicolumn{9}{|l|}{$\begin{array}{l}\text { Propiedad del } \\
\text { recurso }\end{array}$} \\
\hline \multicolumn{9}{|l|}{$\begin{array}{l}\text { Uso actual del } \\
\text { recurso }\end{array}$} \\
\hline $\begin{array}{l}\text { Existencia de } \\
\text { equipamiento } \\
\text { turístico }\end{array}$ & & & & & & & & \\
\hline
\end{tabular}

Fuente: Elaboración propia.

Como se puede apreciar en el tabla No. 1, las variables de localización, calidad del entorno, actividades, propiedad y equipamiento turístico, son integradas por todas las metodologías expuestas, ya que son datos primordiales para el registro de datos de los recursos a evaluar. En cambio, las variables de condiciones generales, periodo de visita, tiempo de recorrido, tipo de visitante y nivel de afluencia, son las que se registran de manera diferenciada. Particularmente se señala que la mayoría de las metodologías solo consideran datos sobre los recursos, no así sobre los visitantes que llegan a ellos.

Cabe aclarar que las metodologías presentan diferencias en cuanto a su funcionalidad para distintos ámbitos. La metodología de la OMT (2001) se considera la más adecuada para inventariar recursos en un ámbito nacional con distintos niveles de jerarquía y consolidación. En un ámbito regional las metodologías de la OEA (2009), Bote (2002) y SECTUR (2009) son las herramientas que mejor se adecuan, ya que se pueden aplicar tanto a recursos potenciales como a atractivos ya en uso, además de ser apropiadas para 
recursos de tipo cultural. Para ámbitos locales, se consideran propicias las metodologías de Zamorano (2002), Godfrey y Clarke (2002), Oscos (1990) y Franco et al. (2009).

De las anteriormente expuestas, en esta investigación se optó por trabajar con la metodología de Franco et al. (2009), en virtud de que es específica para evaluar recursos naturales y contempla tanto la fase de inventario como la de evaluación. Su formulación se fundamenta en la evaluación multicriterio, por tanto ofrece una valoración cuantativa de la potencialidad de los recursos, aportando un resultado objetivo y jerarquizable. Esta metodología ha sido aplicada para espacios naturales locales, como en el caso del Parque Nacional Nevado de Toluca, Estado de México, (Osorio et. al., 2009), y del Parque Estatal Sierra de Nanchititla, Estado de México (Enríquez et al., 2010), en los que ha probado ser una herramienta útil para la evaluación de los recursos y la propuesta de creación de productos turísticos alternativos.

De acuerdo a lo establecido por Franco et.al (2009: 212), la evaluación multicriterio (EMC) "comprende un conjunto de técnicas que permiten evaluar diversas alternativas de elección a la luz de múltiples criterios y prioridades", teniendo como propósito apoyar la toma de decisiones entre las alternativas a elegir. La metodología consiste en la construcción de una matriz que refleja las características de un conjunto dado de alternativas de elección a partir de una serie de criterios.

El conjunto de alternativas se refiere en este caso, al conjunto de recursos o atractivos naturales, culturales o mixtos, caracterizados por una serie de atributos a los cuales se les considera criterios. La matriz expresa las cualidades (valor numérico o simbólico) de la alternativa o unidad de observación con respecto a los " $n$ " atributos considerados (Barba y Pomerol, 1997). Una vez construida la matriz de decisiones es posible aplicar algún procedimiento de evaluación para asignar un valor que refleje la medida en que dicha alternativa cumple con el objetivo planteado en la evaluación. La tabla 2 muestra el procedimiento básico de la EMC.

Tabla 2 - Descripción de los componentes para la valoración de los recursos recreativos turísticos.

\begin{tabular}{|l|l|}
\hline \multicolumn{1}{|c|}{ Componente } & \multicolumn{1}{c|}{ Descripción } \\
\hline $\begin{array}{l}\text { Identificación } \\
\text { del conjunto } \\
\text { de alternativas } \\
\text { posibles. }\end{array}$ & $\begin{array}{l}\text { Depende del objetivo, de la escala del problema (alternativas de decisión) y de la } \\
\text { escala a la cual operan los tomadores de decisiones. }\end{array}$ \\
$\begin{array}{l}\text { Definición del } \\
\text { conjunto de } \\
\text { atributos (objetivos } \\
\text { y atributos } \\
\text { asociados). }\end{array}$ & $\begin{array}{l}\text { Un criterio de evaluación incluye tanto al concepto de atributos como de } \\
\text { objetivos. Un objetivo es un enunciado acerca del estado deseado de un sistema } \\
\text { Geográfico del mundo real e indica la dirección de mejora de uno o más atributos } \\
\text { de un elemento del sistema. La cuantificación de un objetivo es la adopción de } \\
\text { alguna escala que, directa o indirectamente, miden el nivel de logro (atributo). }\end{array}$ \\
\hline $\begin{array}{l}\text { Obtención } \\
\text { de atributos } \\
\text { (normalizados). }\end{array}$ & $\begin{array}{l}\text { La información geográfica suele expresarse en diversas escalas (nominal, } \\
\text { ordinal, de intervalo y de razón) y debe transformarse a una escala común } \\
\text { mediante diversos procedimientos de conversión. La construcción de la matriz } \\
\text { de decisión implica no solamente que las variables se expresen numéricamente, } \\
\text { sino que se encuentren en una misma escala de medida, por ello es preciso } \\
\text { aplicar algún procedimiento de normalización. }\end{array}$ \\
\hline
\end{tabular}




\begin{tabular}{|l|l|}
\hline & $\begin{array}{l}\text { Métodos no compensatorios. El valor de un criterio no puede verse afectado por } \\
\text { el valor de los demás criterios y las alternativas son evaluadas por el conjunto de } \\
\text { criterios sin establecer una interacción entre ellos. } \\
\text { Métodos compensatorios asumen que el rendimiento de una alternativa en } \\
\text { un criterio determinado puede compensarse por su rendimiento en otro u } \\
\text { otros criterios. Uno de los métodos compensatorios más utilizados es el de la } \\
\text { Sumatoria Lineal Ponderada: }\end{array}$ \\
$\begin{array}{l}\text { Combinación } \\
\text { de los atributos } \\
\text { normalizados } \\
\text { y ponderados } \\
\text { utilizando una regla } \\
\text { de decisión para } \\
\text { obtener el puntaje } \\
\text { total para cada } \\
\text { alternativa. }\end{array}$ & $\begin{array}{l}\text { Siendo: } \\
r_{i} \text { el nivel final de cumplimiento del objetivo de la alternativa } \\
i\end{array}$ \\
\hline $\begin{array}{l}w_{j} \text { el peso del criterio } j \\
v_{i j} \text { el valor ponderado de la alternativa i en el criterio } j\end{array}$
\end{tabular}

Fuente: Tomado de Franco et al., 2009.

El procedimiento descrito fue aplicado a los recursos inventariados en el municipio de SPL. La información fue recabada a través de una cédula de observación retomando la utilizada para la evaluación de recursos de las ANP de los Parques del Nevado y Nanchititla. Se inventariaron 8 recursos turísticos en 5 recorridos de campo realizados durante los meses de enero y abril de 2013, clasificándolos en recursos terrestres y acuáticos de acuerdo con sus principales rasgos geográficos. La cédula incluyó siete aspectos fundamentales: datos generales del sitio, localización, características específicas, condiciones generales, información sobre visitantes, observaciones adicionales y evaluación general. Con base en Bote (2002), las variables de análisis que describen las condiciones de uso del recurso como son el acceso, las posibilidades de apreciación, la infraestructura, los servicios, y la seguridad, quedaron referidos como criterios extrínsecos en la metodología; los elementos propios de cada recurso tales como su dimensión, su tipo de vegetación o de claridad de agua, y sus elementos escénicos quedaron considerados como criterios intrínsecos. El inventario obtenido y la evaluación realizada se exponen en el siguiente apartado.

\section{Resultado de la evaluación de los recursos naturales de SPL}

Con base en la metodología EMC descrito en el apartado anterior, se llevó acabo un procedimiento de evaluación que incluyó las siguientes etapas:

a) Definición del objetivo e identificación de alternativas

b) Definición del conjunto de atributos

c) Obtención de la matriz de decisión

d) Valoración de los recursos turísticos. 
A continuación se desarrolla el resultado de cada una de las etapas enunciadas para el caso de estudio.

\section{Identificación de alternativas}

Las alternativas obtenidas a partir del inventario de los recursos fueron 8: un parque ecológico, un árbol emblemático, dos lagunas, dos balnearios, una alberca natural y un río. Su localización, categorías y características se presentan en la Figura. 1 y la Tabla 3.
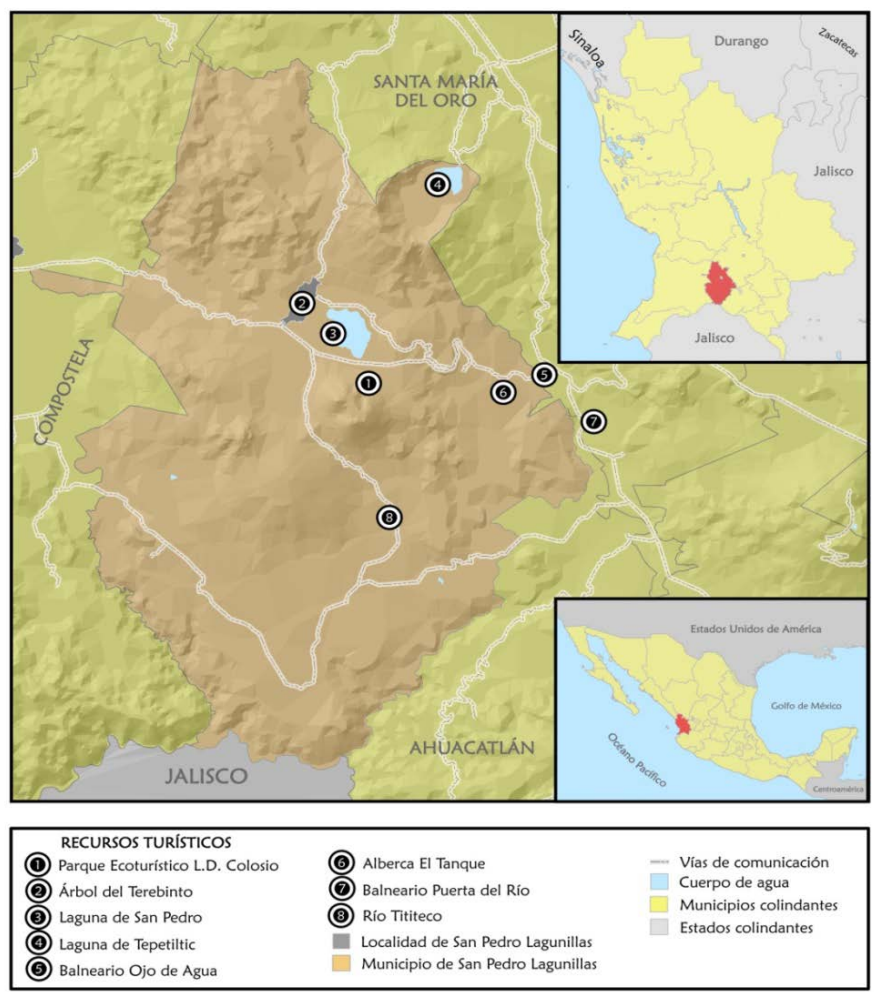

Figura 1 - Recursos Turísticos Naturales de SPL.

Fuente: Mapa elaborado por el Dr. Héctor Hugo Regil García a partir del inventario de recursos naturales de SPL.

Tabla 3 - Recursos Turísticos Naturales del Municipio de San Pedro Lagunillas

\begin{tabular}{|l|l|l|}
\hline 1.- Parque Ecológico Luis Donaldo Colosio \\
\hline Categoría & Sitio Natural \\
\hline Tipo & Terrestre \\
\hline Subtipo & -- \\
\hline Características & $\begin{array}{l}\text { Es un parque que data de } 1998 \mathrm{y} \mathrm{su} \\
\text { característica principal es que se } \\
\text { encuentra conformado por pinos, es } \\
\text { visitado solo por los pobladores del } \\
\text { municipio }\end{array}$ \\
\hline
\end{tabular}




\begin{tabular}{|c|c|c|}
\hline \multicolumn{2}{|c|}{ 2.- Árbol del Terebinto } & \multirow{2}{*}{ 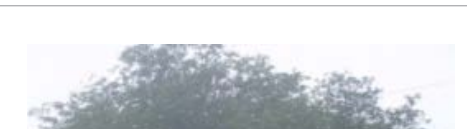 } \\
\hline Categoría & Sitio Natural y Cultural & \\
\hline Tipo & Terrestre & $\therefore x+\frac{1}{3}$ * \\
\hline Subtipo & -- & \\
\hline Características & $\begin{array}{l}\text { Es considerado el primer árbol } \\
\text { de esta especie traído a América } \\
\text { Latina. }\end{array}$ & $=10$ \\
\hline \multicolumn{2}{|c|}{ 3.- Laguna de San Pedro } & \\
\hline Categoría & Sitio Natural & \\
\hline Tipo & Acuático & \\
\hline Subtipo & Laguna & \\
\hline Características & $\begin{array}{l}\text { Es la laguna más importante de } \\
\text { la región, pertenece al programa } \\
\text { turístico de Lagunas Encantadas. }\end{array}$ & \\
\hline \multicolumn{2}{|c|}{ 4.- Laguna de Tepetiltic } & \\
\hline Categoría & Sitio Natural & \\
\hline Tipo & Acuático & \\
\hline Subtipo & Laguna & \\
\hline Características & $\begin{array}{l}\text { Es una pequeña laguna donde } \\
\text { se puede practicar la pesca y la } \\
\text { natación. }\end{array}$ & \\
\hline \multicolumn{2}{|c|}{ 5.- Balneario "Ojo De Agua" } & \\
\hline Categoría & Sitio Natural & \\
\hline Tipo & Acuático & \\
\hline Subtipo & Manantial & \\
\hline Características & $\begin{array}{l}\text { Es un cuerpo de agua que emana } \\
\text { del cerro cercano, la temperatura } \\
\text { del agua es idónea para practicar } \\
\text { natación. }\end{array}$ & (2) \\
\hline \multicolumn{2}{|c|}{ 6. Alberca "El Tanque" } & \\
\hline Categoría & Sitio natural & \\
\hline Tipo & Acuático & \\
\hline Subtipo & -- & \\
\hline Características & $\begin{array}{l}\text { Alberca ejidal, se abre una vez al } \\
\text { año y se llena con agua desviada del } \\
\text { manantial ojo de agua. }\end{array}$ & \\
\hline \multicolumn{2}{|c|}{ 7.-Balneario “Puerta del Río" } & \\
\hline Categoría & Sitio Natural & \\
\hline Tipo & Acuático & \\
\hline Subtipo & Natural & \\
\hline Características & $\begin{array}{l}\text { Se compone de cuatro albercas pero } \\
\text { su atractivo principal es la parte } \\
\text { del rio San Pedro que cruza por la } \\
\text { localidad y es la parte atractiva del } \\
\text { sitio. }\end{array}$ & \\
\hline
\end{tabular}




\begin{tabular}{|l|l|}
\hline 8.- Rio Tititeco & Sitio Natural \\
\hline Categoría & Acuático \\
\hline Tipo & Rio \\
\hline Subtipo & $\begin{array}{l}\text { Se ubica en la comunidad de Amado } \\
\text { Nervo, en el sitio se practica la } \\
\text { pesca y el avistamiento de fauna } \\
\text { endémica. }\end{array}$ \\
\hline Características & \\
\hline
\end{tabular}

\section{Fuente: elaboración propia.}

Las condiciones generales para arribar a los sitios son muy variadas, por ejemplo, existen vialidades pavimentadas para llegar al Árbol del Terebinto y a la Laguna de San Pedro, en cambio para el Balneario Ojo de Agua, la Laguna de Tepetiltic y el rio Tititeco, se llega a través de un camino de terracería y no existe señalización alguna. Cabe mencionar que el gobierno del estado ha invertido para la realización de caminos para llegar a estos sitios y que las obras están a punto de concluir. También se señala que los únicos sitios que poseen infraestructura turística son la Laguna de San Pedro y el Balneario Puerta del Río, en contraste con el Balneario Ojo de Agua y la Alberca El Tanque, que no poseen infraestructura alguna. Una mención adicional es que recursos de gran belleza escénica como el Río Tititeco, a la fecha no ha sido aprovechado turísticamente debido a la inseguridad que priva en la localidad donde se encuentran.

Así entonces, la integración del inventario permitió un acercamiento a la condición de los recursos, identificando que existen recursos de alto potencial como es el caso de la Laguna de San Pedro y el balneario Puerta del Río que sin embargo cuentan con liimitada infraestructura y equipamiento.

\section{Conjunto de atributos}

En esta etapa, se definieron los atributos aplicables a cada tipo de recurso, ya fuera terrestre o acuático, tanto para los criterios intrínsecos como para los extrínsecos, describiendo con precisión los ítems clasificatorios de cada atributo. En la tabla 4 se muestra el conjunto de criterios y atributos para cada tipo de recurso. 
Tabla 4 - Criterios y atributos para la evaluación de recursos turísticos de SPL

\begin{tabular}{|c|c|c|c|c|c|c|}
\hline \multicolumn{4}{|c|}{ Criterios intrínsecos } & \multicolumn{3}{|c|}{ Criterios extrínsecos } \\
\hline $\begin{array}{l}\text { Tipo de } \\
\text { recurso }\end{array}$ & Criterio & Atributo & Descripción & Criterio & Atributo & Descripción \\
\hline \multirow{4}{*}{$\begin{array}{l}\text { Terrestres } \\
\text { (cerros, } \\
\text { barrancas, } \\
\text { parques, } \\
\text { etc.). }\end{array}$} & a) Cobertura & $\begin{array}{l}\text { a1) Vegeta- } \\
\text { ción }\end{array}$ & $\begin{array}{l}\text { Abundante (A) } \\
\text { Suficiente (S) } \\
\text { Escasa (E) }\end{array}$ & \multirow[b]{2}{*}{ g) Acceso físico } & \multirow[t]{2}{*}{ g1) Distancia } & Kilómetros \\
\hline & \multirow[b]{2}{*}{$\begin{array}{l}\text { b) Condiciones } \\
\text { del terreno }\end{array}$} & b1) Gradiente & $\begin{array}{l}\text { Muy inclinado (MI) } \\
\text { Inclinado (I) } \\
\text { Algo inclinado (AI) } \\
\text { Plano (P) }\end{array}$ & & & Minutos \\
\hline & & $\begin{array}{l}\text { b2) Super- } \\
\text { ficie }\end{array}$ & $\begin{array}{l}\text { Pedregosa }(\mathrm{Pe}) \\
\text { Terracería }(\mathrm{T}) \\
\text { Húmeda }(\mathrm{H}) \\
\text { Compacta }(\mathrm{C}) \\
\text { Pastizal }(\mathrm{Pa}) \\
\text { Otra }(0)\end{array}$ & \multirow{5}{*}{$\begin{array}{l}\text { h) Posibilida- } \\
\text { des de aprecia- } \\
\text { ción }\end{array}$} & $\begin{array}{l}\text { h1) Estado de con- } \\
\text { servación }\end{array}$ & $\begin{array}{l}\text { Excelente (E) } \\
\text { Bueno (B) } \\
\text { Regular (R) } \\
\text { Malo (M) }\end{array}$ \\
\hline & & b3) Dificultad & $\begin{array}{l}\text { Alto (A) } \\
\text { Medio (M) } \\
\text { Bajo (B) }\end{array}$ & & $\begin{array}{l}\text { h2) Calidad del } \\
\text { entorno }\end{array}$ & $\begin{array}{l}\text { Optima (0) } \\
\text { Buena (B) } \\
\text { Regular (R) } \\
\text { Baja (Ba) }\end{array}$ \\
\hline \multirow{5}{*}{$\begin{array}{l}\text { Acuáticos } \\
\text { (lagos) }\end{array}$} & \multirow[t]{2}{*}{$\begin{array}{l}\text { c) Caracterís- } \\
\text { ticas }\end{array}$} & $\begin{array}{l}\text { c1) Dimen- } \\
\text { sión }\end{array}$ & Metros & & h3) Interés & $\begin{array}{l}\text { Grande }(G) \\
\text { Alguno }(A) \\
\text { Poco }(P) \\
\text { Ninguno }(N)\end{array}$ \\
\hline & & $\begin{array}{l}\text { C2) Transpa- } \\
\text { rencia }\end{array}$ & $\begin{array}{l}\text { Cristalina (C) } \\
\text { Semiturbia (S) } \\
\text { Turbia (T) }\end{array}$ & & h4) Fragilidad & $\begin{array}{l}\text { Muy alta (MA) } \\
\text { Alta (A) } \\
\text { Moderada (M) } \\
\text { Baja (B) }\end{array}$ \\
\hline & $\begin{array}{l}\text { d) Atractivo } \\
\text { adicional }\end{array}$ & d1) Fauna & $\begin{array}{l}\text { Sí (S) } \\
\text { No (N) }\end{array}$ & & h5) Contaminación & $\begin{array}{l}\text { Sí (S) } \\
\text { No (No) }\end{array}$ \\
\hline & & \multirow{2}{*}{$\begin{array}{l}\text { d2) Proximi- } \\
\text { dad }\end{array}$} & \multirow{2}{*}{$\begin{array}{l}\text { Directo al caudal } \\
\text { (C) } \\
\text { A Distancia (D) }\end{array}$} & \multirow{4}{*}{$\begin{array}{l}\text { i) Infraestructu- } \\
\text { ra y servicios }\end{array}$} & i1) Señalización & $\begin{array}{l}\text { Suficiente (S) } \\
\text { Alguna (A) } \\
\text { Insuficiente (I) } \\
\text { Ninguna (N) }\end{array}$ \\
\hline & & & & & i2) Equipamiento & $\begin{array}{l}\text { Suficiente (S) } \\
\text { Alguno (A) } \\
\text { Insuficiente (I) } \\
\text { Ninguno (N) }\end{array}$ \\
\hline \multirow{4}{*}{$\begin{array}{l}\text { Acuáticos } \\
\text { (corrientes } \\
\text { de agua) }\end{array}$} & \multirow{3}{*}{$\begin{array}{l}\text { e) Caracterís- } \\
\text { ticas }\end{array}$} & e1) Ancho & Metros & & $\begin{array}{l}\text { i3) Instalaciones } \\
\text { recreativas }\end{array}$ & $\begin{array}{l}\text { Instalaciones } \\
\text { recreativas (A) } \\
\text { Elementos } \\
\text { susceptibles } \\
\text { de recreación } \\
(M) \\
\text { Ningún lugar } \\
\text { recreativo (B) }\end{array}$ \\
\hline & & $\begin{array}{l}\text { e2) Transpa- } \\
\text { rencia }\end{array}$ & $\begin{array}{l}\text { Cristalina (C) } \\
\text { Semiturbia (S) } \\
\text { Turbia (T) }\end{array}$ & & i4) Actividades & $\begin{array}{l}\text { No. total de } \\
\text { actividades }\end{array}$ \\
\hline & & $\begin{array}{l}\text { e3) Extensión } \\
\text { visible }\end{array}$ & $\begin{array}{l}1 \text { a } 3 \text { mts. (A) } \\
3 \text { a } 6 \text { mts. (B) } \\
6 \text { a } 10 \text { mts. (C) } \\
\text { Más de } 10 \text { mts. (D) }\end{array}$ & \multirow[t]{2}{*}{ j) Seguridad } & \multirow[t]{2}{*}{ j1) Vigilancia } & \multirow{2}{*}{$\begin{array}{l}\text { Muy seguro } \\
\text { (M) } \\
\text { Seguro (S) } \\
\text { Moderado } \\
\text { (Mo) } \\
\text { Inseguro (I) }\end{array}$} \\
\hline & $\begin{array}{l}\text { f) Atractivo } \\
\text { adicional }\end{array}$ & F1) Fauna & $\begin{array}{l}\text { Sí (S) } \\
\text { No (N) }\end{array}$ & & & \\
\hline
\end{tabular}

Fuente: Tomado de Franco et al. (2009). 
Una vez definidos los atributos fue posible obtener la matriz general de acuerdo a los criterios intrínsecos y extrínsecos para cada recurso. Para su mejor apreciación se presenta dividida la matriz por cada criterio, mostrando los resultados de los criterios intrínsecos en las tabla 5.

Tabla 5 - Caracterización de los criterios intrínsecos de los recursos naturales de SPL.

\begin{tabular}{|c|c|c|c|c|c|c|c|c|}
\hline \multirow{2}{*}{\multicolumn{2}{|c|}{$\begin{array}{l}\text { RECURSO } \\
\text { Terrestres }\end{array}$}} & \multicolumn{7}{|c|}{ CRITERIOS } \\
\hline & & \multicolumn{2}{|c|}{$\begin{array}{c}\text { Vegetación } \\
\text { A1(1) }\end{array}$} & \multicolumn{2}{|c|}{$\begin{array}{l}\text { Gradiente } \\
\text { B1(2) }\end{array}$} & \multicolumn{2}{|c|}{$\begin{array}{c}\text { Superficie } \\
\text { B2(3) }\end{array}$} & $\begin{array}{l}\text { Dificultad } \\
\text { B3(4) }\end{array}$ \\
\hline \multicolumn{2}{|c|}{$\begin{array}{l}\text { Parque Ecológico Luis } \\
\text { Donaldo Colosio }\end{array}$} & \multicolumn{2}{|l|}{$\mathrm{E}$} & \multicolumn{2}{|l|}{$P$} & \multicolumn{2}{|l|}{$\mathrm{Pa}$} & B \\
\hline \multicolumn{2}{|c|}{ Árbol Terebinto } & \multicolumn{2}{|l|}{$\mathrm{E}$} & \multicolumn{2}{|l|}{$P$} & \multicolumn{2}{|l|}{ C } & B \\
\hline \multicolumn{2}{|c|}{$\begin{array}{l}\text { Acuáticos (Lagos y } \\
\text { lagunas) }\end{array}$} & \multicolumn{2}{|c|}{$\begin{array}{l}\text { Dimensión } \\
C_{1}\end{array}$} & \multicolumn{2}{|c|}{$\begin{array}{l}\text { Transparencia } \\
\text { C2 (5) }\end{array}$} & \multicolumn{2}{|l|}{$\begin{array}{l}\text { Fauna } \\
\text { D1 (6) }\end{array}$} & $\begin{array}{l}\text { Proximidad } \\
\text { D2 (7) }\end{array}$ \\
\hline \multicolumn{2}{|c|}{$\begin{array}{l}\text { Laguna de San } \\
\text { Pedro }\end{array}$} & \multicolumn{2}{|l|}{$1.8 \mathrm{Km}$} & \multicolumn{2}{|l|}{$\mathrm{S}$} & \multicolumn{2}{|l|}{$\mathrm{S}$} & $\mathrm{D}$ \\
\hline \multicolumn{2}{|c|}{ Laguna de Tepetiltic } & \multicolumn{2}{|l|}{$1.2 \mathrm{Km}$} & \multicolumn{2}{|l|}{ C } & \multicolumn{2}{|l|}{$\mathrm{N}$} & D \\
\hline \multicolumn{2}{|c|}{ Balneario Ojo de Agua } & \multicolumn{2}{|l|}{$5 \mathrm{~ms}$} & \multicolumn{2}{|l|}{ C } & \multicolumn{2}{|l|}{$\mathrm{N}$} & D \\
\hline \multicolumn{2}{|c|}{ Alberca El Tanque } & \multicolumn{2}{|l|}{$10 \mathrm{~ms}$} & \multicolumn{2}{|l|}{$\mathrm{S}$} & \multicolumn{2}{|l|}{$\mathrm{N}$} & $\mathrm{D}$ \\
\hline \multicolumn{2}{|c|}{ Acuáticos (Ríos) } & \multicolumn{2}{|l|}{$\begin{array}{l}\text { Ancho } \\
\text { E1 }\end{array}$} & \multicolumn{2}{|c|}{$\begin{array}{l}\text { Transparencia } \\
\text { E2 (5) }\end{array}$} & \multicolumn{2}{|c|}{$\begin{array}{l}\text { Extensión visible } \\
\text { E3 }_{3}(9)\end{array}$} & $\begin{array}{l}\text { Fauna } \\
\text { F1 (6) }\end{array}$ \\
\hline \multicolumn{2}{|c|}{ Balneario Puerta del Río } & $8 \mathrm{~ms}$ & & $\mathrm{~S}$ & & $A$ & & $\mathrm{~N}$ \\
\hline Río Tititeco & & $27 \mathrm{~ms}$ & & $C$ & & c & & $\mathrm{S}$ \\
\hline $\begin{array}{l}{[1]} \\
\mathrm{A}= \\
\text { abundante } \\
\mathrm{S}= \\
\text { suficiente } \\
\mathrm{E}=\text { escasa }\end{array}$ & $\begin{array}{l}{[2]} \\
\mathrm{Ml}=\text { muy } \\
\text { inclinado } \\
\mathrm{Al}=\text { algo } \\
\text { inclinado } \\
\mathrm{P}=\text { plano }\end{array}$ & $\begin{array}{l}{[3]} \\
\mathrm{Pe}= \\
\text { pedregosa } \\
\mathrm{C}= \\
\text { compacta } \\
\mathrm{Pa}= \\
\text { pradera }\end{array}$ & $\begin{array}{l}{[4]} \\
A=\text { alto } \\
M=\text { medio } \\
B=\text { bajo }\end{array}$ & $\begin{array}{l}{[5]} \\
C= \\
\text { cristalina } \\
S= \\
\text { semiturbio } \\
T=\text { turbia }\end{array}$ & $\begin{array}{l}{[6]} \\
S=s i \\
N=\text { no }\end{array}$ & $\begin{array}{l}{[7]} \\
D=\text { directo } \\
\text { al caudal } \\
A=a \\
\text { distancia }\end{array}$ & $\begin{array}{l}{[8]} \\
T=\text { Todo el } \\
\text { año } \\
E= \\
\text { Estacional }\end{array}$ & $\begin{array}{l}\text { [9] } \\
1 \text { a } 3 \mathrm{~m}(\mathrm{~A}) \\
\text { 3 a } 6 \mathrm{~m}(\mathrm{~B}) \\
6 \text { a } 10 \mathrm{~m}(\mathrm{C}) \\
\text { Más de } 10 \mathrm{~m}(\mathrm{D})\end{array}$ \\
\hline
\end{tabular}

Fuente: Elaboración propia.

Como se puede observar en la tabla anterior los resultados arrojan que los recursos terrestres tienen una baja dificultad en su acceso, pero poseen escasa vegetación, disminuyendo la posibilidad de su disfrute. En el caso de los recursos actuáticos, la Laguna de Tepetiltic, el Balneario Ojo de Agua y el Río Tititeco cuentan con una aceptable transparencia en el agua, ya que no han sido afectados de manera considerable por la contaminación. Por el atributo de la fauna, los recursos más sobresalientes son la Laguna de San Pedro y el Rio Tititeco, que conservan especies endémicas de peces y aves.

El resultado de la caracterización de los criterios extrínsecos muestra que ninguno de los recursos se presenta de manera destacada en el conjunto de los rubros como se aprecia en la tabla 6. 
Tabla 6 - Caracterización de los criterios extrínsecos de los recursos naturales de SPL.

\begin{tabular}{|c|c|c|c|c|c|c|c|c|c|c|c|c|}
\hline \multirow{3}{*}{ RECURSO } & \multicolumn{12}{|c|}{ CRITERIOS } \\
\hline & \multicolumn{2}{|c|}{ Acceso físico } & \multicolumn{6}{|c|}{ Posibilidades de apreciación } & \multicolumn{3}{|c|}{$\begin{array}{l}\text { Infraestructura } \\
\text { y servicios }\end{array}$} & \multirow{2}{*}{$\begin{array}{l}\text { Seguridad } \\
J_{1}(8)\end{array}$} \\
\hline & $\mathrm{G}_{1}$ & $\mathrm{G}_{2}$ & $\mathrm{H}_{1}(1)$ & $\begin{array}{l}\mathrm{H}_{2} \\
(2)\end{array}$ & $\begin{array}{l}\mathrm{H}_{3} \\
(3)\end{array}$ & $\begin{array}{l}\mathrm{H}_{4} \\
(4)\end{array}$ & $\begin{array}{l}\mathrm{H}_{5} \\
(5)\end{array}$ & $11(6)$ & $\begin{array}{l}12 \\
(6)\end{array}$ & $\begin{array}{l}13 \\
(7)\end{array}$ & 14 & \\
\hline $\begin{array}{l}\text { Parque Ecológico Luis } \\
\text { Donaldo Colosio }\end{array}$ & $57 \mathrm{~km}$ & 7omin & M & $\mathrm{R}$ & A & A & $\mathrm{s}$ & A & A & A & 4 & Mo \\
\hline Árbol Terebinto & $57 \mathrm{~km}$ & 7omin & E & 0 & G & M & $\mathrm{N}$ & $\mathrm{S}$ & $\mathrm{s}$ & M & 2 & M \\
\hline $\begin{array}{c}\text { Acuáticos (lagos y } \\
\text { lagunas) }\end{array}$ & $\mathrm{G}_{1}$ & $\mathrm{G}_{2}$ & $\mathrm{H}_{1}(1)$ & $\mathrm{H}_{2}(2)$ & $\mathrm{H}_{3}(3)$ & $\mathrm{H}_{4}(4)$ & $\mathrm{H}_{5}(5)$ & $\mid 1(6)$ & $12(6)$ & $13(7)$ & 14 & $\mathrm{~J} 1(8)$ \\
\hline Laguna de San Pedro & $57 \mathrm{Km}$ & $50 \mathrm{~min}$ & $\mathrm{R}$ & B & $A$ & M & $\mathrm{S}$ & $\mathrm{S}$ & A & M & 3 & $\mathrm{~S}$ \\
\hline Laguna de Tepetiltic & $50 \mathrm{Km}$ & 4omin & B & B & A & A & $\mathrm{N}$ & $\mathrm{N}$ & $\mathrm{N}$ & B & 3 & $\mathrm{~s}$ \\
\hline Balneario Ojo de Agua & $55 \mathrm{~km}$ & $50 \mathrm{~min}$ & $\mathrm{~B}$ & B & A & B & $\mathrm{N}$ & $A$ & I & B & 2 & M \\
\hline Alberca El Tanque & $55 \mathrm{~km}$ & $50 \mathrm{~min}$ & $\mathrm{R}$ & B & $\mathrm{P}$ & B & $\mathrm{N}$ & 1 & 1 & B & 2 & M \\
\hline Acuáticos (Ríos) & $\mathrm{G}_{1}$ & $\mathrm{G}_{2}$ & $\mathrm{H}_{1}(1)$ & $\mathrm{H} 2(2)$ & $\mathrm{H}_{3}(3)$ & $\mathrm{H}_{4}(4)$ & $\mathrm{H}_{5}(5)$ & $11(6)$ & $12(6)$ & $13(7)$ & 14 & $J_{1}(8)$ \\
\hline Balneario Puerta del Río & $60 \mathrm{~km}$ & 6omin & B & B & A & M & $\mathrm{s}$ & A & $\mathrm{S}$ & A & 4 & $\mathrm{~s}$ \\
\hline Rio Tititeco & $77 \mathrm{~km}$ & $120 \mathrm{~min}$ & E & 0 & A & B & $\mathrm{N}$ & $\mathrm{N}$ & $\mathrm{N}$ & B & 5 & 1 \\
\hline $\begin{array}{c}{[1]} \\
\mathrm{E}=\text { excelente } \\
\mathrm{B}=\text { bueno } \\
\mathrm{R}=\text { regular } \\
\mathrm{M}=\text { malo } \\
{[8]} \\
\mathrm{M}=\text { muy seguro } \\
\mathrm{S}=\text { seguro } \\
\mathrm{Mo}=\text { moderado } \\
\mathrm{I}=\text { inseguro }\end{array}$ & $\begin{array}{l}{[2]} \\
\mathrm{O}=\mathrm{opt} \\
\mathrm{B}=\mathrm{bue} \\
\mathrm{R}=\mathrm{reg} \\
\mathrm{Ba}=\mathrm{ba}\end{array}$ & & $\begin{array}{l}{[3]} \\
G=g r \\
A=a l g \\
P=\text { po } \\
N=\text { nir }\end{array}$ & $\begin{array}{l}\text { nde } \\
\text { uno } \\
\text { o } \\
\text { guno }\end{array}$ & & $\begin{array}{l}{[4]} \\
M a=m b \\
\text { alta } \\
A=\text { alta } \\
M=\text { moc } \\
\text { rada } \\
B=\text { baja }\end{array}$ & & $\begin{array}{l}{[5]} \\
S=s i \\
N=\text { no }\end{array}$ & & $\begin{array}{l}{[6]} \\
\mathrm{S}=\text { su } \\
\text { ciente } \\
\mathrm{I}=\text { Ins } \\
\text { ciente } \\
\mathrm{A}=\mathrm{al} \\
\mathrm{N}=\mathrm{ni}\end{array}$ & $\begin{array}{l}\text { fi- } \\
\text { ufi- } \\
\text { suna } \\
\text { nguna }\end{array}$ & $\begin{array}{l}{[7]} \\
A=\text { instalacio- } \\
\text { nes recrea- } \\
\text { tivas } \\
M=\text { elemen- } \\
\text { tos suscep- } \\
\text { tibles de } \\
\text { recreación } \\
B=\text { ningún } \\
\text { lugar recre- } \\
\text { ativo }\end{array}$ \\
\hline
\end{tabular}

Fuente: Elaboración propia.

Se destaca que el Balneario Puerta del Río es el único dotado con equipamiento e instalaciones recreativas. El Río Tititeco es el único que presenta un excelente estado de conservación y no muestra elementos contaminantes, sin embargo es el recurso que se ubica a mayor distancia de la capital estatal.

\section{Matriz de decisión}

Una vez tabulados los valores de los criterios, fue posible llevar acabo su transformación a una escala de entre 1 y 10. El valor de cada atributo representa el grado en que cada alternativa cumple con el objetivo de valoración, siendo 10 el valor máximo. Con base en esto fue posible construir la matriz de decisión que se presenta en la tabla 7 . 
Tabla 7 - Matriz de decisión con valores normalizados.

\begin{tabular}{|c|c|c|c|c|c|c|c|c|c|c|c|c|c|c|c|c|c|}
\hline \multirow{2}{*}{$\begin{array}{l}\text { Recursos } \\
\text { Terrestres }\end{array}$} & \multicolumn{16}{|c|}{ Atributos } & \multirow{2}{*}{ SUM } \\
\hline & va1 & vb1 & $\mathrm{vb} 2$ & vb3 & vg1 & vg2 & vh1 & vh2 & $v_{3}$ & vh4 & vh5 & vi 1 & vi 2 & vi 3 & vi 4 & vj 1 & \\
\hline $\begin{array}{l}\text { Parque ecológico Luis } \\
\text { Donaldo Colosio }\end{array}$ & 1 & 10 & 10 & 10 & 4 & 5 & 1 & 3 & 7 & 3 & 5 & 3 & 7 & 10 & 8 & 4 & 91 \\
\hline Árbol del Terebinto & 1 & 10 & 5 & 10 & 4 & 5 & 10 & 10 & 10 & 7 & 10 & 10 & 10 & 8 & 7 & 10 & 127 \\
\hline $\begin{array}{l}\text { Acuáticos (Lagos y } \\
\text { Lagunas) }\end{array}$ & vC1 & VC2 & vd1 & $\mathrm{vd} 2$ & vg1 & vg2 & vh1 & vh2 & vh3 & vh4 & vh5 & vi 1 & vi 2 & vi 3 & vi 4 & vj 1 & \\
\hline Laguna de San Pedro & 10 & 5 & 10 & 10 & 4 & 5 & 4 & 6 & 6 & 6 & 1 & 10 & 5 & 5 & 5 & 6 & 98 \\
\hline Laguna de Tepetiltic & 8 & 10 & 5 & 10 & 10 & 10 & 6 & 6 & 6 & 4 & 10 & 1 & 1 & 1 & 5 & 6 & 99 \\
\hline Balneario Ojo de Agua & 3 & 10 & 5 & 10 & 6 & 7 & 6 & 6 & 6 & 10 & 10 & 4 & 6 & 1 & 3 & 10 & 103 \\
\hline Alberca el tanque & 5 & 5 & 5 & 10 & 6 & 7 & 4 & 6 & 4 & 10 & 10 & 6 & 6 & 1 & 3 & 10 & 98 \\
\hline Acuáticos (Ríos) & ve1 & ve2 & ve3 & $\mathrm{vf}_{1}$ & vg1 & $\operatorname{vg} 2$ & vh1 & vh2 & vh3 & vh4 & vh5 & vi 1 & vi 2 & vi 3 & vi 4 & vj 1 & \\
\hline Balneario Puerta del Rio & 3 & 5 & 10 & 10 & 6 & 3 & 6 & 6 & 6 & 6 & 5 & 4 & 10 & 10 & 8 & 6 & 104 \\
\hline Rio Tititeco & 10 & 10 & 4 & 5 & 1 & 1 & 10 & 10 & 6 & 1 & 6 & 1 & 1 & 1 & 10 & 1 & 78 \\
\hline
\end{tabular}

Fuente: Elaboración propia con base en Franco et. al. (2009).

Al realizar una sumatoria simple de los valores normalizados se obtiene que el Árbol del Terebentino obtiene la mayor calificación con un resultado de 127 puntos, seguido del Balneario Puerta del Río con 104 puntos y del Balneario Ojo de Agua con 103 puntos. El Río Tititeco fue el recurso con menor puntaje, no obstante los destacados atributos que lo distinguieron en la etapa anterior.

Por ultimo se realizara la valoracion de los recursos turisticos como se muestra enseguida.

\section{Valoración de los recursos turísticos}

Una vez construida la matriz de decisión, se aplicó el procedimiento de combinación lineal ponderada. Para ello se requiere la definición de los valores de ponderación de los criterios, diferenciando la importancia relativa de cada uno de los criterios. En seguimiento a la metodología elegida, se otorgó un peso de $40 \%$ a los criterios intrínsecos y un 60\% para los criterios extrínsecos, obteniendo el resultado total ponderado que aparece en la tabla 8. 
Tabla 8 - Resultados ponderados de los criterios.

\begin{tabular}{|c|c|c|c|c|c|}
\hline Recurso & $\begin{array}{l}\text { Suma lineal } \\
\text { sin } \\
\text { ponderación }\end{array}$ & $\begin{array}{c}\text { Suma ponderada } \\
\text { de Criterios } \\
\text { extrínsecos }\end{array}$ & $\begin{array}{c}\text { Suma ponderada } \\
\text { de Criterios } \\
\text { intrínsecos }\end{array}$ & Valor final & $\begin{array}{c}\text { Orden de } \\
\text { preferencia }\end{array}$ \\
\hline $\begin{array}{l}\text { Parque Ecológico } \\
\text { Luis Donaldo } \\
\text { Colosio }\end{array}$ & 91 & 5.93 & 2.56 & 8.49 & 1 \\
\hline Árbol del Terebinto & 127 & 3.83 & 2.26 & 6.09 & 5 \\
\hline $\begin{array}{l}\text { Laguna de San } \\
\text { Pedro }\end{array}$ & 98 & 3.02 & 3.68 & 6.7 & 4 \\
\hline $\begin{array}{l}\text { Laguna de } \\
\text { Tepetiltic }\end{array}$ & 99 & 4.09 & $3 \cdot 32$ & 7.41 & 2 \\
\hline $\begin{array}{l}\text { Balneario Ojo de } \\
\text { Agua }\end{array}$ & 103 & 1.58 & 2.84 & 4.42 & 7 \\
\hline Alberca el Tanque & 98 & 4.22 & 2.72 & 6.94 & 3 \\
\hline $\begin{array}{l}\text { Balneario Puerta } \\
\text { del Rio }\end{array}$ & 104 & $3 \cdot 3$ & 2.74 & 6.04 & 6 \\
\hline Rio Tititeco & 78 & 1.43 & 2.89 & $4 \cdot 32$ & 8 \\
\hline
\end{tabular}

Fuente: Elaboración propia con base en Franco et. al. (2009)

Como se puede apreciar, el recurso que obtiene la mejor puntuación (8.49) fue el Parque Ecológico Luis Donaldo Colosio, debido a que tuvo el mayor puntaje en la suma ponderada de criterios extrínsecos (5.93). La Laguna de San Pedro resultó ser el recurso que obtuvo la mayor calificación en la suma ponderada de los criterios intrínsecos (3.68), aunque su posición desciende hasta un cuarto lugar debido al resultado de la suma de sus criterios extrínsecos (3.02).

A partir del valor final obtenido en la evaluación, se resuelve que el Parque Ecológico Luis Donaldo Colosio, la Laguna Tepetitic, la Alberca el Tanque y la Laguna de San Pedro son elegibles para detonar la actividad turística, por lo que pueden conformar un producto recreativo-turístico que atraiga el desplazamiento de visitantes potenciales.

\section{Propuesta de producto turístico en spl}

En virtud de que el lugar de estudio tiene potencial para el desarrollo de un turismo alternativo, se propone la creación de un producto turístico de aventura, el cual es propicio para incentivar los viajes basados en actividades recreativas asociadas con desafíos impuestos por la naturaleza, buscando el beneficio de las comunidades del entorno. (SECTUR, 2008). En el producto de turismo de aventura se tomarán en cuenta actividades como caminata, ciclismo, kayaquismo y tirolesa, ya que las condiciones de los recursos evaluados son propicias para llevarlas a cabo. Con fundamento en el modelo de producto turístico realizado por Rodríguez et al. (2013), en la tabla 9 se expone la propuesta del producto a crearse en SPL. 
Tabla 9 - Propuesta de producto turístico de aventura para SPL.

\section{PRODUCTO DE TURISMO DE AVENTURA EN EL MUNICIPIO DE SAN PEDRO LAGUNILLAS}

Imagen a promover: Naturaleza, actividades deportivas y al aire libre, lagunas, montañas y bosque.

Producto: Excursionismo y turismo de aventura.

Actividades a considerar: ciclismo, kayakismo, caminatas, senderismo interpretativo, circuito de tirolesa, campo de gotcha, gastronomía local.

Objetivo y Metas:

- Objetivos: generar un producto turístico de calidad para el desarrollo del municipio de SPL.

- Metas: corto plazo (1 año) creación de una cartera de proyectos y propuestas para su desarrollo; mediano plazo (3-5 años) poner en marcha los proyectos; largo plazo (10 años) consolidar el producto en la región como uno de los más importantes de turismo de naturaleza.

Recursos

- Parque ecológico Luis Donaldo Colosio

- Laguna de Tepetiltic

- Alberca El Tanque

- Laguna de San Pedro

\section{Accesos y Tiempos}

Tiempo de llegada al Parque Ecológico Luis Donaldo tomando como punto de partida Tepic viajando en automóvil: $70 \mathrm{~min}$.

Costo de recorrido: $\$ 150$

Tiempo aproximado: $6 \mathrm{hrs}$.

\section{Estrategias generales:}

- Ordenamiento y sustentabilidad ecológica: controlar el uso de suelo y de los cuerpos de agua para el disfrute sustentable de los sitios y que el turista sea parte del cuidado ambiental.

- Límite de carga aceptable: establecer cuál será el número de visitantes que permanecerán en cada recurso, esto con el fin de preservar cada espacio en óptimas condiciones.

- Manejo sustentable de energía: utilización de celdas solares para generar la electricidad que se requiere en los sitios cerrados y capacitación a los pobladores para su manejo.

- Manejo sustentable de agua: fomentar el cuidado responsable del agua entre los visitantes y pobladores instalando llaves ahorradoras y captación de agua de lluvia.

- Manejo de residuos sólidos: separación y reciclaje de residuos (implementación de rellenos sanitarios y separación de desechos).

- Guías locales: establecer rutas específicas para cada actividad, el personal encargado debe estar capacitado y certificado para dar el servicio requerido.

- Zonas de descanso con mapas que indiquen su posición dentro del circuito.

- Información al visitante y capacitar al personal para dar mejor atención al visitante.

\section{Acciones específicas para la puesta en marcha del producto}

Limpieza de las zonas, acondicionamiento de caminos, diseño de rutas, adaptación de estacionamientos, construcción de baños y vestidores, instalación y compra de equipo para tirolesa, kayakismo y gotcha, contratación de personal, acondicionamiento y colocación de zonas de descanso, construcción de establecimientos de alimentos y bebidas.

Capacitación sobre: administración de servicios, calidad de servicio, uso debido de equipo para tirolesa, kayakismo y gotcha, primeros auxilios y seguridad.

\section{Mercado objetivo}

Perfil: Familias, adolescentes y jóvenes entre 12 y 25 años.

Segmento: Familias de nivel socioeconómico medio-medio alto, escuelas, grupos y asociaciones deportivas.

Experiencias para el visitante: Práctica de deportes extremos, contacto con la naturaleza, senderismo, natación y ejercicio físico. 
El producto que se propone tomará al Parque Luis Donaldo Colosio - recurso con la mayor valoración obtenida - como el punto de partida para generar un circuito turístico que incluya la visita a la Laguna de Tepetiltic, a la Alberca El Tanque y a la Laguna de San Pedro.

\section{Conclusiones}

Como herramientas de apoyo para la toma de decisiones, las metodologías de evaluación de recursos turísticos resultan útiles en tanto desvelan los atributos de valor de cada uno de ellos. Como se expuso en el texto, existen varias opciones para llevar a cabo dicho ejercicio, pero es claro que su elección corresponde al ámbito de estudio, ya sea nacional, estatal o local.

No obstante la riqueza descriptiva y analítica que otorgan las metodologías cualitativas, para esta investigación se consideró pertinente la valoración cuantitativa de los recursos que se logra con la aplicación de la metodología de evaluación multicriterio, ya que disminuyó la subjetividad de la evaluación, permitió obtener datos duros ponderados a partir de los criterios de análisis y otorgó fundamento a la jerarquización resultante.

De acuerdo a la sumatoria ponderada de los criterios intrínsecos y extrínsecos aplicada a los recursos de SPL, el Parque Ecológico Luis Donaldo Colosio, la Laguna Tepetiltic, la Alberca El Tanque y la Laguna de San Pedro, son los recursos que pueden conformar un producto turístico de "despegue" de la actividad turística en este municipio, proyectando una imagen de turismo de naturaleza que se concrete en un conjunto de experiencias de aventura. El resto de los recursos, particularmente los de carácter acuático, pueden fungir como recursos complementarios que paulatinamente se integren en circuitos cortos en torno al producto turístico base.

Finalmente, es necesario señalar que la factibilidad de que los recursos sean puestos en valor a través de la conformación de uno o varios productos turísticos, está sujeta a las posibilidades de inversión, a la cultura ecológica y a la disposición de las comunidades aledañas, para abrir la oportunidad de que en el futuro el municipio sea conocido con un importante abanico de atractivos turísticos dentro de la región.

\section{Referencias}

BARBA, S.; POMEROL, J. Decisiones multicriterio. Fundamentos teóricos y utilización práctica. Colección Economía, Universidad de Alcalá, Alcalá de Henares. 1997.

BOTE, V. Planificación económica del turismo de una estrategia masiva a una artesanal. Trillas. México. 2002.

BOULLÓN, R. Los Planes de Manejo. Aportes y Transferencias, v. 10, p. 35-42. 2006.

ENRÍQUEZ, M. et al. Evaluación multicriterio de los recursos turísticos del Parque Estatal Sierra de Nanchititla, Estado de México. El Periplo Sustentable, n. 18, p. 7-35. 2010.

FRANCO, S. et al. Evaluación multicriterio de los recursos turísticos: Parque Nacional Nevado de Toluca - México. Estudios y Perspectivas en Turismo, v. 18, n. 2. p. 208-226, 2009. 
GARZÓN, R.; ARIAS, E. La Planificación y Ordenación del Uso Público en Espacios Protegidos Andaluces: Contextualización Global y Análisis Específico en La Sierra Norte de Sevilla. Cuadernos de Turismo, n. 21, p. 33-65. 2008.

GOBIERNO DE LA REPÚBLICA. Programa Sectorial de Turismo. Plan Nacional de Desarrollo 2013-2018. Ed: Gobierno de la República, México. 2013.

GODFREY, K.; CLARKE, J. The tourism development handbook. London, Ed: Continuum. 2000.

H. AYUNTAMIENTO DE SAN PEDRO LAGUNILLAS. Plan de Desarrollo Municipal 2007-2012 San Pedro Lagunillas. Ed: H. Ayuntamiento de San Pedro Lagunillas, Nayarit, México. 2007.

INEGI. Censo de población y vivienda 2010. Disponible en: 〈http://www.inegi.org.mx/est/contenidos/ proyectos/ccpv/cpv2010/default.aspx> Acceso: 6 dic. 2013.

JIMÉNEZ, A. Turismo: estructura y desarrollo. McGraw- Hill. México.1993

ORGANIZACIÓN DE ESTADOS AMERICANOS (OEA). Metodología de Inventario Turístico. OEA, México. 2009.

ORGANIZACIÓN MUNDIAL DEL TURISMO (OMT). Apuntes de metodología de la investigación del Turismo. OMT. Madrid, España. 2001.

OSCOS, J. Metodología para la elaboración de inventarios de atractivos turísticos. Tesis. Facultad de Turismo de la UAEM. Toluca, México. 1990.

OSORIO, M. et al. Programa Turístico Sustentable del Parque Nacional Nevado de Toluca, México. Reporte Final. UAEM, México. 2009.

RAMÍREZ, I.; OSORIO, M. "Propuesta teórico-metodológica para la planeación del turismo sustentable en el Parque Iztaccíhuatl-Popocatépetl”, en Maribel Espinosa Castillo, Tendencias de investigación turística a principios del siglo XXI. Instituto Politécnico Nacional, México, p. 217-243. 2007.

.Propuesta de desarrollo recreativo-turístico para el Parque Nacional Iztaccíhuatl-Popocatépetl. In: OSORIO, M.; CASTILLO, M. Entorno del turismo, v. 3. Universidad Autónoma del Estado de México, p. 173-208. 2008.

RODRÍGUEZ, V. et al. Planificación del desarrollo recreativo-turístico sustentable en el ejido de la Peñuela, Parque Nacional Nevado de Toluca. Teoría y Praxis, n. 14, p. 25-58. 2013.

SECTUR. Estudio Estratégico de Viabilidad del Segmento de Ecoturismo en México, Resumen Ejecutivo. Centro de Estudios Superiores en Turismo (CESTUR), México. 2001.

SECTURa. Guía para la presentación de proyectos ecoturísticos. Disponible en: 〈http://www.cdi.gob. $\mathrm{mx} /$ ecoturismo/docs/guia_presentacion_proyectos_semarnat.pdf>Acceso en: 14 nov. 2013.

SECTURb. Guía de apoyos federales para el desarrollo de proyectos de turismo de naturaleza. Disponible en: 〈http://moodle2.unid.edu.mx/dts_cursos_mdl/lic/AET/TR/AM/o2/Apoyos_Turismo. pdf>. Acceso en: 14 nov. 2013.

TORRE, M. G.; BOSCHI, A. M. Planificación y Gestión de la Recreación en Áreas Protegidas. Anuario de Estudios en Turismo, año 4, v. III, p. 45-61. 2014.

WORLD ECONOMIC FORUM. The Travel \& Tourism Competitiveness. Report 2015. Ed: World Economic Forum. 2015.

ZAMORANO, F. Turismo alternativo, servicios turísticos diferenciados. Trillas. México, 2012. 